\title{
Biometric Recognition for Pet Animal
}

\author{
Santosh Kumar, Sanjay Kumar Singh \\ Department of Computer Science \& Engineering, I.I.T. (BHU), Varanasi, India \\ Email: santosh.rs.cse12@iitbhu.ac.in, sks.cse@iitbhu.ac.in
}

Received 26 March 2014; revised 20 April 2014; accepted 28 April 2014

Copyright (C) 2014 by authors and Scientific Research Publishing Inc.

This work is licensed under the Creative Commons Attribution International License (CC BY). http://creativecommons.org/licenses/by/4.0/

(c) (i) Open Access

\begin{abstract}
Missing, swapping, false insurance claims and reallocation of pet animals (dog) are global problems throughout the world and research done to solve this problem is minimal. Traditional biometrics and non-biometrics methods have their own boundaries and they fail to provide competent level of security to pet animal (dog). The work on animal identification based on their phenotype appearance (coat patterns) has been an active research area in recent years and automatic face recognition for dog is not reported in the literature. Dog identification needs innovative research to protect the pet animal. Therefore it is imperative to initiate research, so that future face recognition algorithm will be able to solve this important problem for identification of pet animal (like dog, cat). In this paper an attempt has been made to minimize the above mentioned problems by biometrics face recognition of dog. The contributions of this research are: 1) implementation of an existing biometrics algorithm which mitigates the effects of covariates for dogs; 2) proposed fusion based method for recognition of pet animal with $94.86 \%$ accuracy. Thus in this paper, we have tried to demonstrate that face recognition of dog can be used to recognize the dog efficiently.
\end{abstract}

\section{Keywords}

\section{Animal Biometrics, Pet Animal, Face Recognition, Dog Feature Covariates}

\section{Introduction}

Dogs were the first pet animal to be domesticated in our society and have shared a common environment with humans for over ten thousand years. Dog (canis familiaris) plays a significant role in protection of increased number of industries and different organizations that exploit the behavior of domestic dog. In 2001, there estimated to be 400 million dogs in the world [1]. According to [2] about 7 million animal enter United States and animal shelters every year, only 10 percent make it back to their owners. Body marker system of pet animals such as tattooing, ear notching, and paint/dye can easily get lost; such methods are not enough to provide robustness to animal identification and making it open to missing, swapping, theft, duplication and fraudulence. Dog identification based on biometric traits is a major research issue in livestock management. The accuracy of 
traditional identification method of pet animal such as permanent (Tattoo, Microchip) semi-permanent and temporary (RFID) methodologies are not robust to provide significant level of security to pet animal. These traditional biometrics method and non-biometrics techniques have their own boundaries and they fail to provide competent level of security to pet animal (dog). However, these algorithms may not yield good results and require a large database of pet dog. Thus, there is a need to develop a robust biometric identification system for identifying individual pet animal (dog). Biometrics offers a natural and reliable solution to certain aspects of identity management by utilizing fully automated or semi-automated schemes to recognize individuals (human or animal) based on their inherent physiological and/or behavioral characteristics (traits) or animal's phenotypic appearance [3]. This is an emerging research field of animal detection and identification based on animal biometric trait known as phenotype. A phenotype is a combination of an organism's observation characteristics or traits such as its morphology, biochemical and physiological behavior research field in computer vision and pattern recognition has repeatedly been recognized as an intellectual frontier in different applications (like pet animal and human biometrics) whose boundaries of applicability are yet to be determined. One of such novel applications is known as visual animal biometrics [3].

Visual animal biometric system is basically a pattern recognition based system. It acquires biometric data from an individual, extracts a salient feature set from the data, compares feature set against the feature set(s) stored in the database, and executes an action based on the result of the comparison [3]. Visual animal biometrics plays an important role in multiple disciplines like biometrics, biography, ecology population, computer vision and behavioral research. Visual animal biometric system utilizes both the variability and uniqueness of vocalizations, body dynamics, coat patterns and their morphologies as biometrics traits [3].

The objective of pet dog's recognition is to emphasize on compressive studies of pet animal (dog) identification, traceability and tracking system as a mechanism to stop the pet animal prowling, missing, false claims and theft of pet animal conflicts between owner and imposter or across border between different countries. Pet animal identification system plays an important role in controlling, vaccination management, disease outbreak, their production management, assignment of ownership and dog traceability. On the other hand, using the animal biometric system faces big challenges with respect to identification accuracy, robustness as animal's body dynamic and their body morphological traits may be easily controlled. Driven from this need, the earliest contribution of this innovative research is to gather a database of live captured pet dog which operates as a benchmark for the proposed dog identification scheme.

The overall outline of this paper is as follows: Section 2 demonstrates the literature review, previous existing face recognition method and provides new research work in the area of dog (pet dog) recognition. Section 3 illustrates datasets preparation and proposed algorithm for dog face recognition. Section 4 demonstrates the methodology that is used in detail and Experimental Result. Section 5 explains the experimental performance and its analysis. Section 6 explains conclusion and future direction.

\section{Literature Review}

The visible identification methods such as sketching of pattern [4] ear notches or collars are widely used to discriminate pet animals that have been sterilized, vaccinated and treated as part of a pet animal population management program. This can have the additional benefit of indicating to the community that these animals are being cared for as part of a management program, raising awareness of the organization or authority carrying out the intervention and potentially enhancing public cooperation. The previous means of identification can be summarized and identification methods demonstrate the recognition of pet animal below [4]-[6] (Table 1):

Some methods may be less visible or not visible at all, but enable the identification of individual animal namely tattoos and microchips. These methods enable much more useful data to be recorded during the course of an intervention, enhancing our understanding of the dynamics of stray dog populations. Individual identification can also generate data for a comprehensive population survey enabling more effective monitoring and evaluation. These methods do, however, need to be used in combination with visible methods of identification.

\section{Animal Face Detection and Classification}

Yamada Akihiko et al. proposed methodology for dog and cat face detection method for digital camera base directional edge detection technique [7] Automatic animal identification technologies assist logistic service providers to improve effectiveness and efficiency throughout the logistic process. Both traditional bar code and 2-D 
Table 1. List of animal identification method.

\begin{tabular}{cccccccccc}
\hline $\begin{array}{c}\text { Identification method/ } \\
\text { Attribute }\end{array}$ & \multicolumn{4}{c}{ Permanent Identification Method } & \multicolumn{2}{c}{$\begin{array}{c}\text { Semi-Permanent } \\
\text { Identification Method }\end{array}$} & $\begin{array}{c}\text { Temporary } \\
\text { Identification Method }\end{array}$ \\
\cline { 2 - 9 } & Tattooing & Microchip & Ear-Tip/Notch & Freeze Brand & ID Collar & Ear Tagging & Paint/Dye & RFID \\
\hline Reliability & Medium & Very high & High & Very high & Low & low & Low & Very low \\
Cost & Medium & Very high & Low & Medium & Low & low & Very low & Very low \\
Visibility & Very low & NA & Medium & High & Very high & Very high & Very high & NA \\
Longevity & High & Very High & Very High & High & Low & Low & Very low & Low \\
Risk of harm & Low & Very low & Medium & Low & High low & Very high & Very low & Very low \\
Accuracy & High & Very High & NA & Low & High & Low & Very low & High \\
Uniqueness & High & Very High & NA & Low & High & Medium & Very low & Very High \\
Database Required & High & Very High & NA & Low & Low & medium & NA & Very High \\
\hline
\end{tabular}

symbols based systems can be easily produced by printing. A printed tag and sensor must have line of sight for the data to be able to be read. RFID technology overcomes this limitation [8]. Parkhi, O.M. et al. evaluated the object categorization problem of determining the breed of animal from their image. The object classification approach applied as the task of discriminating the 37 different breeds of pets [9]. Chanvichitkul, M. et al. proposed methodology for dog breed classification or identification based on the dog face images [10]. Furthermore, Genetic testing between dogs and their wolf ancestors propose that canines were first domesticated between 35,000 and 100,000 years ago. V. L. Voithi et al. [11] demonstrated that the large disparities between visual and DNA breed identification as well as differences among people visual identifications of dogs [12]. They evaluated good accuracy of the dog identification by an adoption agency as having specific breeds in their ancestry did not have all of those breeds detected by DNA analysis [13]. However, Genetic testing of pet animal is very costly and time consuming process.

\section{Datasets Preparation and Proposed Algorithm for Dog Face Recognition}

The pet dog images were taken from Dogster, Catster [14] two other social web sites dedicated to the collection and discussion of images of pets from Flickr [15], mydogspace [16] and Google images [17]. Groups and People are uploading images to Catster and Dogster provides the breed information as well and the Flickr groups are unambiguous to each breed, which make things easier tagging. For each of the 50 dog breeds as subject about 300 - 400 images were downloaded from these dog data sources and to form a pool of candidates for inclusion in the dataset. The most general problem in all the dog data sources has no name labels of dog. But it was initiated to be errors in the breed labels. Therefore these labels were re-evaluated by the human annotators and fixed whenever possible. When fixing was not possible, for instance because the pet was a cross breed, the image was dropped. Some images from database are shown in Figure 1. Another challenge is recognizing twins pet animal (dog). Once twins grow up, they may develop differentiating looks but newly born twins are extremely similar (Figure 2).

The feature extraction algorithm such as PCA (principal Component Analysis) [18], LDA (Local Discriminant Analysis) [19] and ICA (Independent Component analysis) [20] are motivated by the observation that dogs have been distinguished (not apparently) facial profiles. Further, it is difficult to restrict pose and huge texture variations of dog, implying that holistic face recognition algorithms may not yield good results. On the other hand, local feature based algorithm may provide good results. These algorithms would work based on covariates independent of conditions during image acquisition process, thus providing equal weightage to each subject up for analysis.

The schematic descriptions of proposed pet animal (dog) face recognition biometrics system can be viewed as having modules: 1) Sensor module; 2) Feature extraction module; 3) Matching module (training and testing phase). In sensor module, pet animal (dog) face image is captured by a suitable sensor device of camera (high resolution) as raw biometric data. In feature extraction module: The quality of the pet animal (dog) biometric data acquiesced by the sensor or camera is first assessed in order to determine its suitability for further processing. After quality of biometric data enhancement, biometric data is then processed and a set of salient discriminatory features extracted by holistic appearance approach (PCA, LDA, ICA and its variants algorithm) 


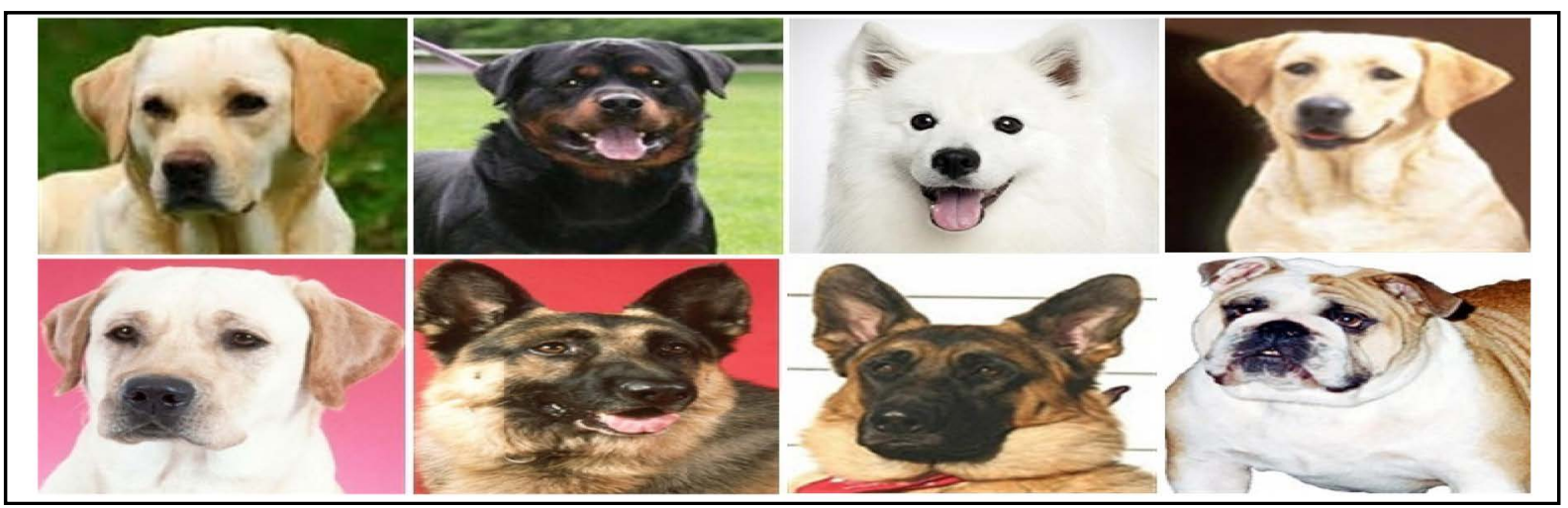

Figure 1. Some images from dog face database.

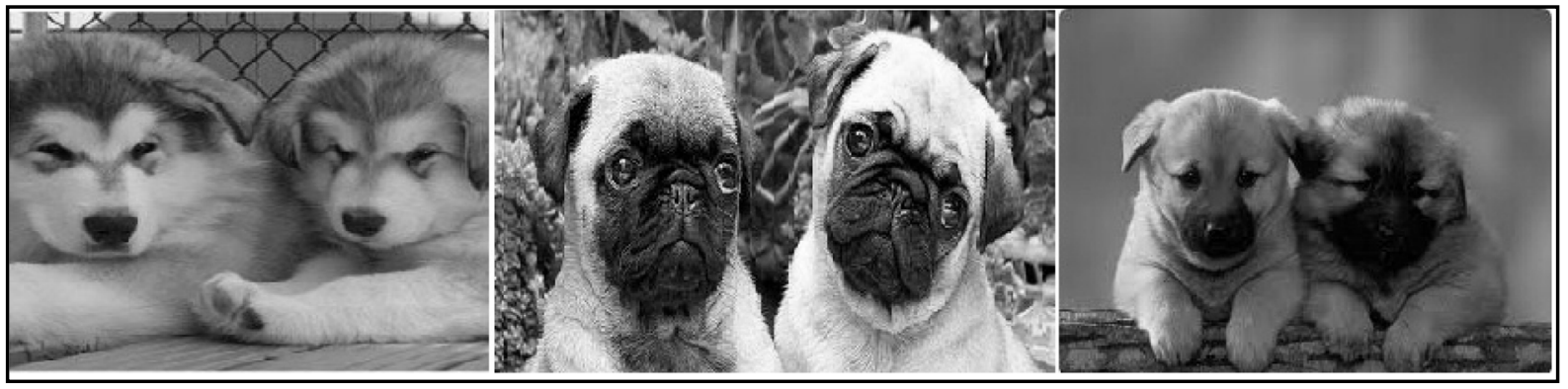

Figure 2. Sample images of twins dog from the dog face database.

and extracted features are normalized and unique discriminative features are selected and stored in template and stored in enrollment template database. As mentioned earlier, the relevant information present in image changes with pose and body dynamics. There are some blurriness and pose variation and preserve good quality of dog images without covariates. These artifacts may be removed by applying the Gaussian (pyramid) smoothing level technique up to four levels. The effect of such artifacts is completely filtered while preserve the discriminative appearance based feature. To extract feature from the original dog face image and four levels Gaussian smoothed images, PCA (Principal Component Analysis), LDA (Local Discriminant Analysis), ICA (Independent Component Analysis) and their modified algorithms (batch_ccipca, ICA, ind_ccipca, isvm, LDA, lda_libsvm, LIBSVM and PCA, PCA_ lisvm) are applied. It also provides motivation to researchers to study their pose, illumination, and matching of their morphological traits. Problem of their body dynamics study can be solved by proposed face recognition methodology. In matching module, face database is divided into two parts: 1) In training phase, system is trained and extracted features from $40 \%$ of pet animal (dog) face database and; 2) In testing phase, it is matched or test against $60 \%$ of database of stored templates to generate match scores and indentifying the pet animal (dog).

The steps involved in pet animal identification block diagram with Gaussian smoothing techniques are illustrated with (Level: 1 dog image dimension (96 × 112)), (Level: 2 dog image dimension (46 × 56)), (Level: 3 dog image dimension $(23 \times 28)$ ) and (Level: 4 dog image dimension $(12 \times 14))$ respectively four levels of Gaussian smoothing are applied so that it can adequately noise filtered while preserving discriminating texture (appearance based feature) information are shown in Figure 3 (Gaussian pyramid level 1). Proposed pet animal (dog) face recognition biometrics system are shown in Figure 4.

\section{Experimental Result}

The performance of proposed biometrics system of pet animal's face recognition is evaluated on the collected dog face (image) database from Google image, dogster and mydogspace. The experimental result of dog face recognition demonstrated in following parts: 1) Experimental performance evaluation; 2) Experimental Analysis. 


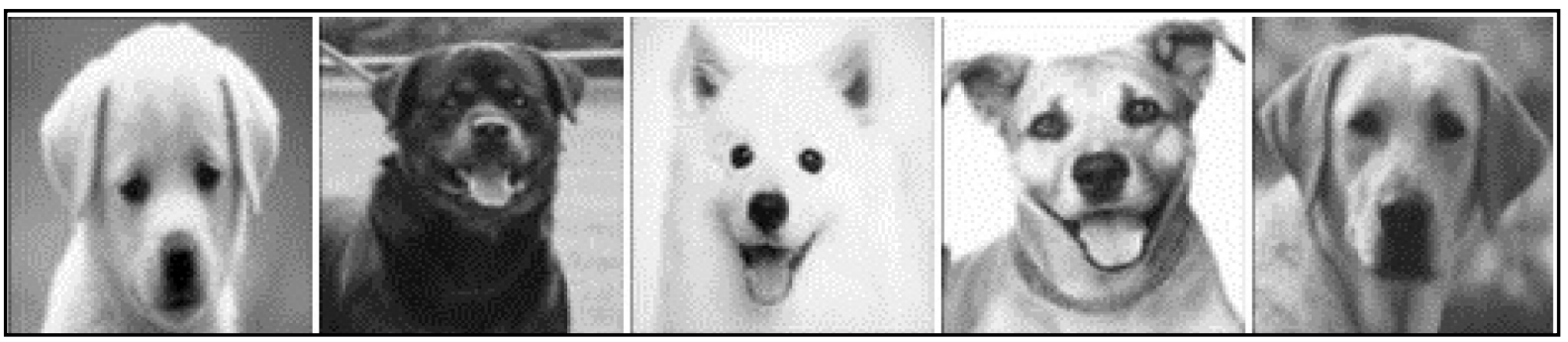

Figure 3. Dog face image of Level: 1 image dimension $(96 \times 112)$.

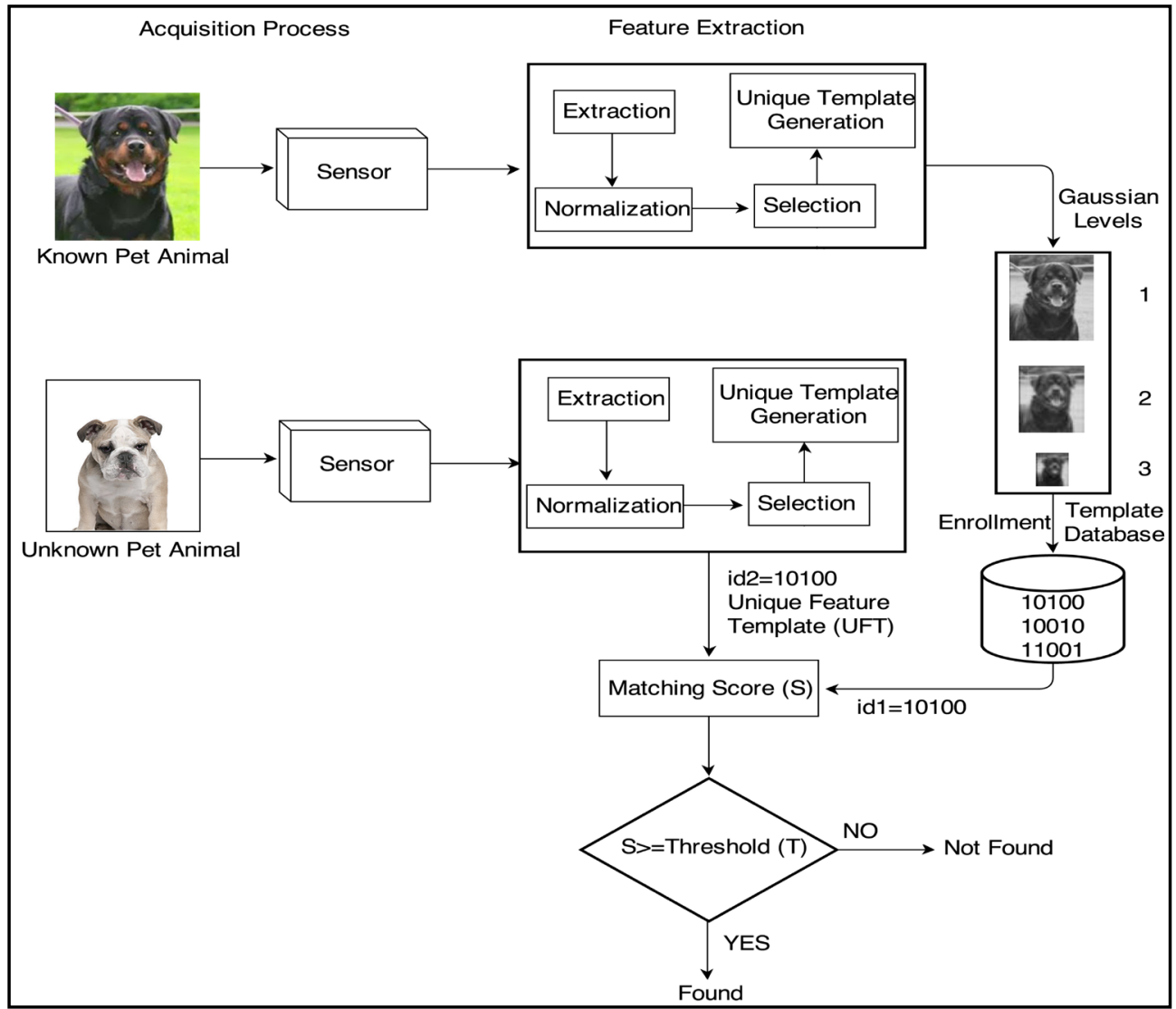

Figure 4. Block diagram for face recognition algorithm for pet animal (dog).

\subsection{Experimental Performance Evaluation}

For experimental result evaluation, the database of dog face (images) was segmented into two parts: 1) training (gallery) part 2) test (probe) part. Six images of each subject (dog) was randomly selected for training part (total of 40 subject $\times 10$ images per subject) and the remaining 160 images were used as probe (test). The training and testing partitioning was performe completed by adding new dog image and updates the old Eigen value $d$ five times for cross validation and rank-1 identification accuracies were computed. The performance evaluation of dog face database has been completed with help of appearance based algorithms (PCA, ICA, LDA), Local Binary Pattern (LBP) [21] [22] and its modified version algorithms (batch-ccipca, independent_ccipca, LDAlibsvm, PCA-lisvm, Batch_ilda, and ilda-libsvm) using our customized version of available source code. The appearance based algorithms and local texture based algorithm (LBP) used for comparison are:

\subsubsection{Principal Component Analysis (PCA)}

Principal component analysis is an unsupervised leaning method and Eigen face which is based on PCA. It was 
invented by Turk and Pentland in 1991and main concept takes a face as a column vector of pixels $X$ where the length of the vector $d$ represents the product of patch's width and height [23]. PCA reduces the dimensionality of each dog face image by make use of similarities between all dog face images. This approach extracts holistic facial feature a set of Eigen faces based on dog face image that combine linearly $Y=A X$ in form of co-variance matrix and describe all dog face image. For given $N$ dog's face image are arranged in column vectors $\Gamma_{1}, \Gamma_{2}, \Gamma_{3} \cdots \Gamma_{N}$ and average face $\bar{N}=\frac{1}{N} \sum_{n=1}^{N} \Gamma_{n}$ is subtracted from each image $\left[\Phi_{i}\right]=\left(\Gamma_{i}-\bar{N}\right)$ (Figure 5) and combine face images into a matrix $\Phi=\left[\Phi_{1}, \Phi_{2}, \Phi_{3} \cdots \Phi_{N}\right]$ is known as co-variance matrix. Principal component analysis finds a set of orthonormal vectors that best representation of face database. These vectors are the eigenvectors $\mu_{k}$ of the covariance matrix $[C]=A A^{T}$. In Eigen space terminology, each face image is projected by the top $N^{\prime}$ significant eigenvector $\mu_{k}$ get weight of top $w_{k}=u_{k}(\Gamma-\bar{N})$ that best linearly weight the Eigen faces into a representation of the original image (Figure 6). Knowing the weights of the training images and a new test face image, a nearest neighbor approach determines the identity of the face. Eigen faces has the advantage of being simple and fast at the cost of low accuracy when pose, expression, and illumination vary significantly.

\subsubsection{Linear Discriminant Analysis (LDA) and Incremental LDA}

Linear Discriminant Analysis is a supervised learning based classification method used for face recognition. It is used classifying samples of unknown classes based on training samples with known classes. This technique aims to maximize between-class (i.e., across users) variance and minimize within-class (i.e., within user) variance. One of the failings in PCA and ICA is that the distances between weights from faces of the same person are greater than face weights from different people. To correct this and find more dog face identification accuracy, a method called Fisherfaces [19] [23]. It attempts to attempts to find vectors that not only describe the data, but also best discriminate between classes of data. Let $C$ class be the dog face with mean of class $j$ denoted by $N_{j}$ and the $i^{\text {th }}$ image in class $\mathrm{j}$ denoted as $x_{i}^{j}$ a between and within class matrix is calculated. These scatter matrix are $s_{b}$ and $s_{w}$ respectively and calculated as follows:

$$
\begin{gathered}
S_{b}=\sum_{j=1}^{C}\left(\bar{N}_{j}-N\right)\left(\bar{N}_{j}-N\right)^{T} \\
S_{w}=\sum_{j=1}^{C} \sum_{i=1}^{N j}\left(x_{i}^{j}-\bar{N}_{j}\right)\left(x_{i}^{j}-\bar{N}_{j}\right)^{T} \\
W_{\text {Frisher-Descriminat }}=\arg \max \frac{\left|W^{T} S_{b} W\right|}{\left|W^{T} S_{w} W\right|}
\end{gathered}
$$

In Linear descriminat analysis, a set of projection vectors $(W)$ is created by using these scatters matrices $\left(S_{b}\right.$ and $S_{w}$ ) are used to maximize the between-class measure while concurrently it minimizing the within-class measure. Literature reviews shows that LDA is repeatedly superior to principal component analysis and it is well for distributed classes in databases which are small [23]. Because LDA approach needs drastically more computation than PCA for large datasets, it is required to explore the use of incremental LDA (ILDA) [24], which is also very efficient in batch mode.

\subsubsection{Independent Component Analysis (ICA)}

Independent component analysis (ICA) is generalized form of PCA and based on non-ortho-normalization of vectors, which allows higher-order dependencies in image pixels to be exploited. It removes the first order (average of database) and second order of database. It is speeded up by including a "sphering" step prior to learning. The row means of $X$ are subtracted and then $X$ is passed through the whitening matrix $W_{z}$, which is twice the inverse square root2 of the covariance matrix $W_{z}=2 \times \operatorname{Cov}(X)^{-1 / 2}$. ICA finds statistically independent images represented by the rows in matrix $\mathrm{V}$ mixed together with $\mathrm{W}$ and denoted by $U=W X$. When the input image to independent component analysis is sphere data, the full transformation matrix WI is produced of sphering matrix and the matrix leads lead by ICA. MacKay and Pearlmutter demonstrated that the ICA approach converges to 


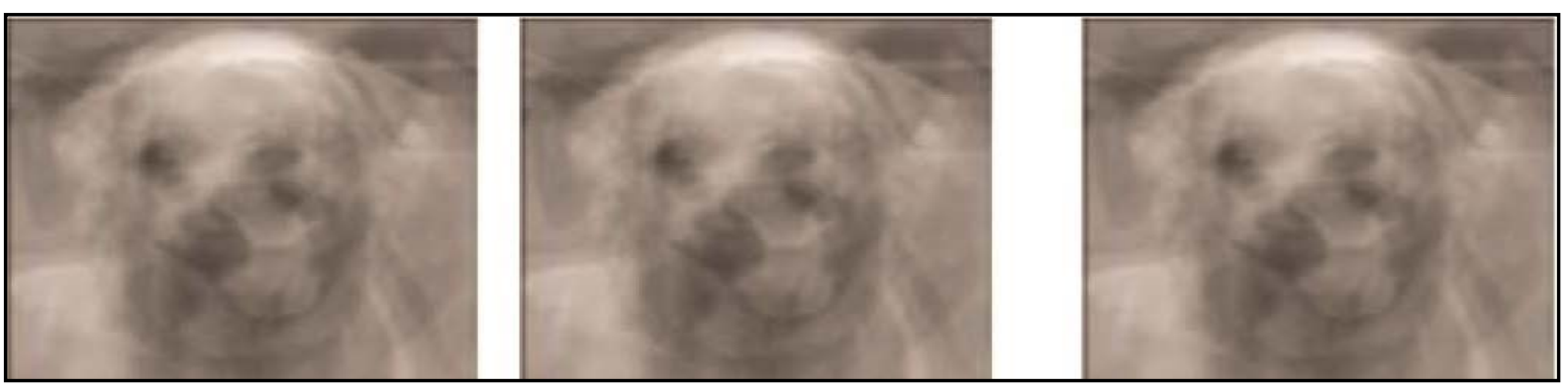

Figure 5. Average value of dog face database.

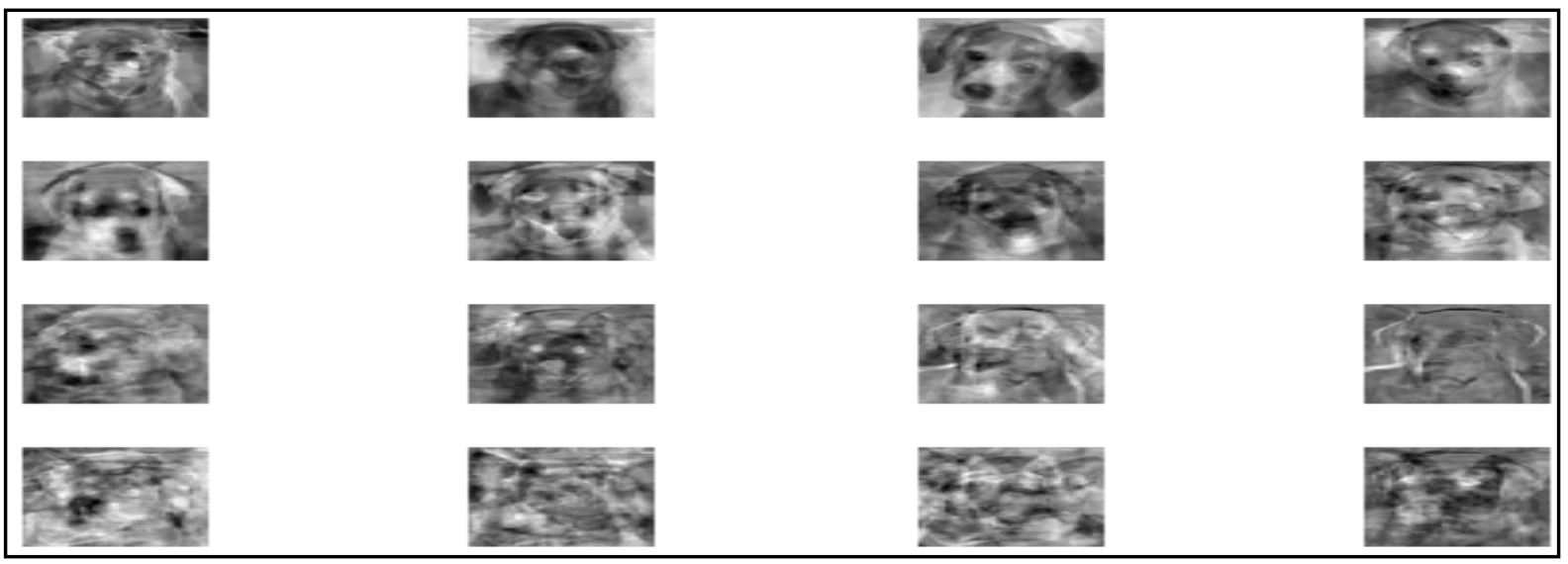

Figure 6. Eigen value of 20 dog face image.

the maximum likelihood estimate of $W-1$ for the generative model of the data: $X=w^{-1} S$ where $S=\left(S_{1}, S_{2}, \cdots S_{N}\right)$ are vectors of impendent component of independent component analysis.

\subsubsection{The Candid Covariance-Free Incremental PCA (CCIPCA) Approach}

The candid covariance-free IPCA (CCIPCA) is a face recognition approach and used to compute the princial components of a sequence of samples or datasets incrementally without estimating the covariance matrix. Thus is known as free covariance-free IPCA approach [24]. It keeps the scale of observations and evaluates the mean (average) of observations in incremental approach for real-time applications and thus it does not take iterations. The characteristic of CCIPCA converges very fast for high dimensional image vectors of data sets. The CCIPCA approach generates observations in a complementary space for the computation of the higher order principal components [25].

\subsubsection{LIBSVM: A Library for Support Vector Machines}

Support vector machine is a popular machine learning algorithm for classification and regression of datasets. [26]. It has achieved much attention for their applicability in solving pattern recognition and computer vision problems. SVMs compute the support vectors through determining a hyper plane that maximizes the margin, or distance between the hyper plane and the closest points [26]. Heisele, B. et al. described in [27], the problem begins with a set of $\mathrm{N}$ points. Each point is labelled as one of two classes $y_{i} \in[1,-1]$ and the best or optimal separating hyper plane $\sum_{i=1}^{l}(w) \times x+b$ where $w=a_{i} y_{i} x_{i}$. It is defined as $\mathrm{N}$ where the sign of $f(x)$ determines the class of the data. For the non-separable case and solving for the coefficients $a_{i}$ and $b$ refer to [28]. The hyper plane in canonical form must satisfy the following constraints $\min _{x_{i}}\left[y_{i}\left(w \cdot x_{i}\right)+b\right] \geq 1, i=1,2,3 \cdots l$ and distance from a hyper plane is given by $d(w, b ; x)=\left|\frac{w \cdot x+b}{w}\right|$. 


\subsubsection{Local Binary Pattern}

Local Binary Pattern (LBP) is a nonparametric descriptor, which competently reviews the local structures of images. It is widely used texture operator of local feature because of its robustness to gray level varies and high computational efficiency. The general LBP descriptor provides a discrete value to a pixel by thresholding a $3 \times 3$ neighborhood window of pixels with the center pixel value $(C)$ and considering the result as a binary number representation. Local Binary Pattern representation of a given pet animal (dog) face image is generated by dividing the image into rectangular grids and computing histogram to measure the frequency of local binary values within each grid. The concatenation of all these histograms constructs the image signature. A version of LBP basic algorithm is to have the neighboring pixels well separated on a circle around a central pixel. The circle can have different radius $(R)$ and varying number of neighbors $(p)$ to account for textures of image at different scales. The circular LBP (CLBP) texture descriptor is computed similarly by thresholding the gray level intensity of pixels with the pixel value of the center of the circle [21] [22]. If the gray level intensity of neighboring pixel is higher or equal, the value is set to one otherwise zero.

$$
\begin{aligned}
& C_{N, R}(p, q)=\sum_{i=0}^{N-1} F\left(n_{i}-n_{c}\right) \times 2^{i} \\
& \text { where } F(.)=\left\{\begin{array}{lr}
1 & \text { if }\left(n_{i}-n_{c}\right) \times 2^{i} \\
0 & \text { otherwise }
\end{array}\right.
\end{aligned}
$$

where $n_{c}$ corresponds to the gray level intensity of center pixel of the circle and $n_{i}$ matches to the gray level intensities of $N$ evenly spaced pixels on a circle of radius $R$. Discriminative power of circular LBP face features favors its use in local region description. Moreover, circular LBP is a local descriptor, thus it is fast to compute and robust to pose, illumination and expression changes. Following are identification accuracy of holistic and texture based face algorithms:

The performance of appearance based algorithms is evaluated at four levels of Gaussian smoothing. The goal of this research emphasizes on various factors which affect recognition performance during dog face database partitioning. It may require novel statistical approach and demonstrates an empirical understanding of which covariates make harder or easier. It may also focus to determine whether and to what degree, a covariate or arrangement of covariates manipulates the presentation of face recognition algorithm. For this intention, we have taken facial expression images in probe and gallery image of dogs. We explored the effect of different facial expression on face recognition performance.

\subsection{Experimental Performance Evaluation}

The experimental analysis of face recognition of pet animal (dog) has been completed with help basic appearance based face recognition algorithms such as PCA (principal component analysis), LDA (local discriminative analysis), ICA (independent component analysis), modified version of PCA, LDA, ICA algorithms and Local Binary Pattern (LBP) texture algorithm. The identification accuracy of dog face (images) has been shown in above Tables 2-5 respectively. The performance of Local Binary Pattern (LBP) algorithm yields the best accuracy of $94.86 \%$ at level 4 in Figure 7 . It was observed that the performance of LBP increases with increasing the levels of Gaussian smoothing. This demonstrates that LBP gives better performance with dog face (images) down sampled by applying Gaussian Pyramid. The performance of ICA yields identification accuracy 89.95\% which is greater than PCA, LDA identification accuracy because it can account for more variation in the input pet animal (dog) face image compared with PCA and LDA.

The Figure 8 illustrates the face recognition performance of PCA and batch candid-covariance free incremental PCA, Independent-CCIPCA, ICA and LDA algorithm respectively. The identification accuracy of PCA $(89.87 \%)$ is higher than other method because it has training dataset compared to the batch-CCIPCA and ICA. ICA get better the accuracy (Table 2) of straight principal component analysis by significantly increasing the computation times and memory requirements in Figure 5. However, when discarding the top 10 Eigen faces of pet animal (database) and ensure sufficient training images are existed in training database (40\% of database) and pet animal (dog) as subject with $<10$ images were discarded, the identification accuracy of PCA giving better, perhaps because the top PCA Eigen faces encode illumination variations. For real time application of pet animal biometrics (identification of the pet animal) scenario has an incremental training process, where identification may be completed by adding new dog image and updates the old Eigen value (Figure 6) and Eigen vec- 


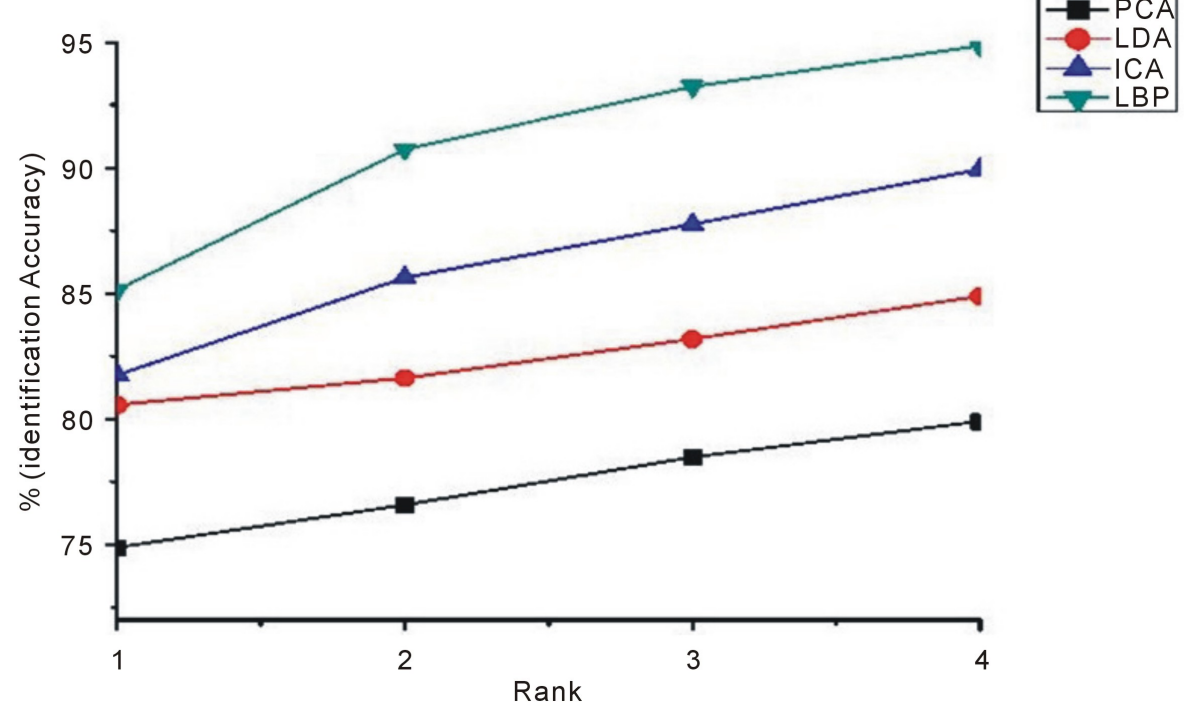

Figure 7. CMC for appearance based face recognition algorithm (PCA, LDA and ICA and LBP (Table 1)).

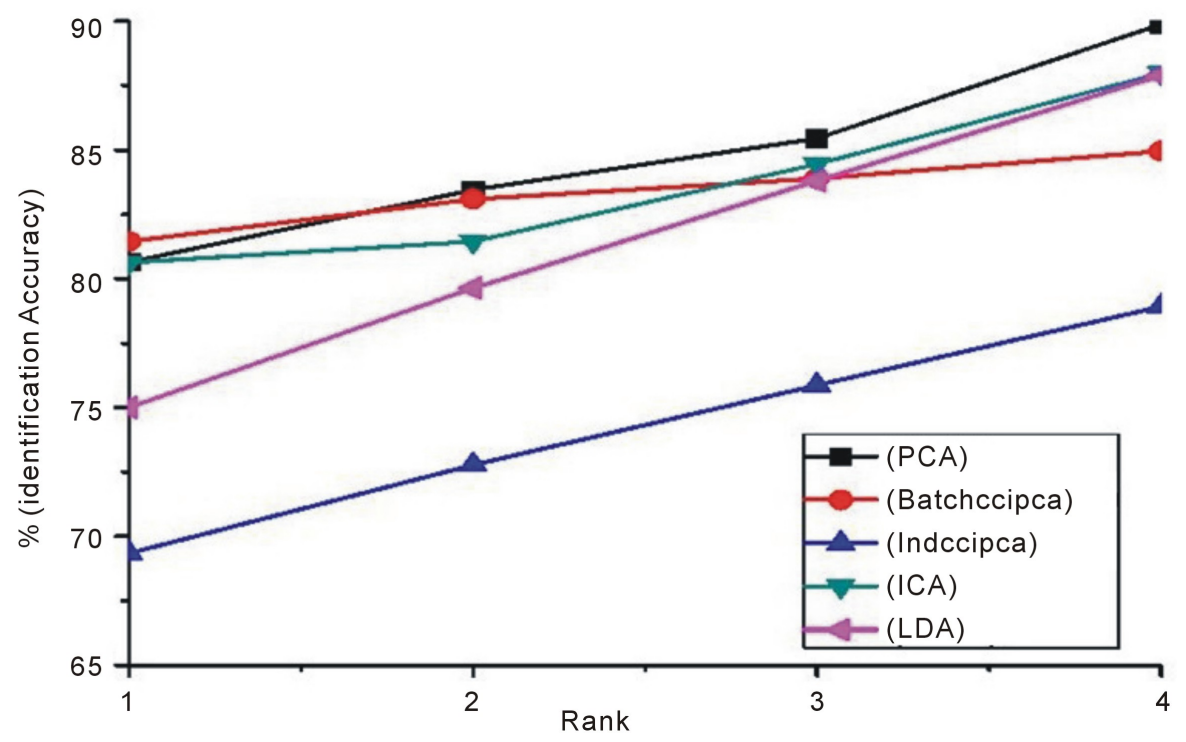

Figure 8. CMC for modified version of appearance based face recognition algorithm (Table 3).

Table 2. Identification accuracy appearance based algorithms: PCA, LDA, ICA and Local Binary Pattern (LBP).

\begin{tabular}{ccccc}
\hline $\begin{array}{c}\text { Gaussian smoothing } \\
\text { Level }\end{array}$ & FCA & Face recognition accuracy (\%) & LBP \\
\hline 1 & 74.89 & LDA & ICA & 85.15 \\
2 & 76.6 & 80.57 & 81.75 & 90.75 \\
3 & 78.5 & 83.19 & 85.64 & 93.25 \\
4 & 79.9 & 84.9 & 87.75 & 94.86 \\
\hline
\end{tabular}

tor by adding new training images (dog). For enhancement of online training Eigen values, we chose to explore covariance free incremental principal component Analysis (CCIPCA) algorithm. In Figure 9, identification accuracy of CCIPCA is $86.3 \%$ than other methods (batch-LDA, ICA-LIBSVM, ILDA and ILDA-LIBSVM) be- 
cause CCIPCA is incremental training approach and adding new dog image and updates the old Eigen value repeatedly so dog database size increases and all LDA invariants (iLDA, ILDA-LIBSVM and batch LDA approaches are not performer on the large datasets. The Figure 9 and Figure 10 demonstrate that face recognition performance based SVM (LIBSVM, PCA-LIBSVM, incremental SVM (iSVM). Identification accuracy of Libsvm is $84.5 \%$ which is greater than PCA-LIBSVM, L DA-LIBSVM and iSVM because LDA, PCA face recognition algorithms are working properly on small datasets and performance of PCA-LIBSVM, LDA-SVM and iSVM are not giving good, decreases at rank-1 and it does not work on large database. Thus we conclude from the experimental results that is giving the highest identification accuracy for pet animal (dog).

\section{Conclusion and Future Direction}

Missing, swapping, false insurance claims of pet animal are global problem throughout the world and exploitation of biometrics and non-biometrics methodologies do not provide the satisfactory level of security to control this problem. These researches establish a new unconstrained dog face database with different face pose varia-

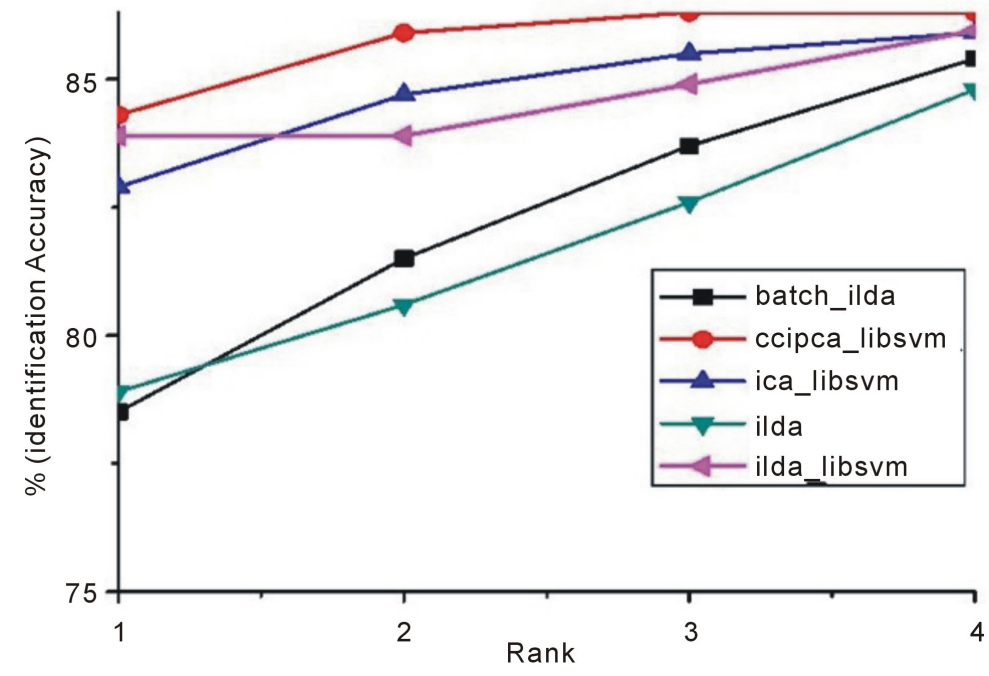

Figure 9. CMC for Intelligent approach with appearance based face recognition algorithm (Table 4).

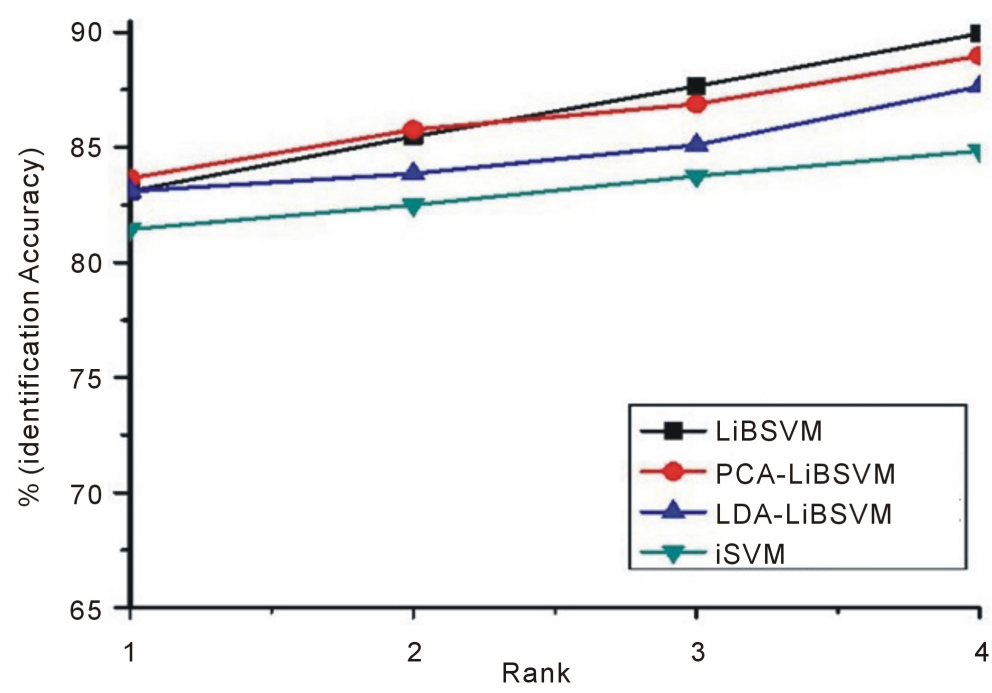

Figure 10. CMC for intelligent approach with appearance based face recognition algorithm (Table 5). 
Table 3. Identification accuracy of PCA, batch_ccipca, ind_ccipca, ICA, LDA algorithms.

\begin{tabular}{cccccc}
\hline \multirow{2}{*}{$\begin{array}{c}\text { Gaussian } \\
\text { smoothing level }\end{array}$} & PCA & batch_ccipca & ind_ccipca & ICA & LDA \\
\cline { 2 - 7 } & 80.65 & 81.45 & 69.35 & 80.64 & 75.0 \\
2 & 83.45 & 83.10 & 72.77 & 81.45 & 79.64 \\
3 & 85.45 & 83.89 & 75.87 & 84.45 & 83.80 \\
4 & 89.87 & 84.95 & 78.93 & 87.98 & 87.89 \\
\hline
\end{tabular}

Table 4. Identification accuracy of batch_ilda, ccipca_libsvm, ica_libsvm, Ilda and ilda_libsvm algorithms.

\begin{tabular}{|c|c|c|c|c|c|}
\hline \multirow{2}{*}{$\begin{array}{c}\text { Gaussian } \\
\text { smoothing Level }\end{array}$} & \multicolumn{5}{|c|}{ Face recognition accuracy (\%) } \\
\hline & batch_ilda & ccipca_libsvm & ica_libsvm & ilda & ilda_libsvm \\
\hline 1 & 78.5 & 84.3 & 82.5 & 78.9 & 83.9 \\
\hline 2 & 81.5 & 85.9 & 84.7 & 80.6 & 83.9 \\
\hline 3 & 83.7 & 86.3 & 85.5 & 82.6 & 84.9 \\
\hline 4 & 85.4 & 86.3 & 85.9 & 84.8 & 85.95 \\
\hline
\end{tabular}

Table 5. Identification accuracy of LIBSVM, PCA-LIBSVM, LDA-LIBSVM and iSVM algorithms.

\begin{tabular}{ccccc}
\hline \begin{tabular}{c} 
Gaussian smoothing $\begin{array}{c}\text { Face recognition accuracy (\%) } \\
\text { level }\end{array}$ \\
\cline { 2 - 5 }
\end{tabular} & LIBSVM & PCA-LIBSVM & LDA-LIBSVM & 83.10 \\
\hline 1 & 83.10 & 83.66 & 83.87 & 81.45 \\
2 & 85.48 & 85.78 & 85.10 & 83.76 \\
3 & 87.67 & 86.88 & 87.65 & 84.85 \\
4 & 89.94 & 88.98 & \multicolumn{2}{c}{} \\
\hline
\end{tabular}

tions. The motivation of this research is to develop new algorithms and provide security to pet animal with the help of biometrics using face recognition. The proposed methodology for identification of pet animal provides high accuracy of $94.86 \%$. Thus, we conclude that face recognition of the dogs can be done in a friendlier, costeffective way if the performances of automatic best matching algorithms are satisfactory. So, it is of vital importance to begin research efforts in this direction to enable future face recognition systems to be able to address the problem of identification of pet animal (dog). Contrary to popular belief that all pet dogs look alike (twin dog identification), the current research provides important insight in identification of dogs. The dog face database has run through proposed face recognition methodologies and has yielded best possible results.

In future, we plan to do further research keeping in view of the following areas. Size of dog face database is to be improved and different environments may be considered throungh acquisition/preparation of dog image for each subject: pose variation, distance variation and illumination variation, occlusion (covering, non-covering) variation. In future, further research may include following:

- Dogs are extremely non-cooperative user of biometrics so it should focus on designing and developing a pose and expression invariant algorithm.

- Covariates-based fusion techniques can be developed as dogs covariates are to be estimated from the pair of images being compared with.

- Illumination variation is a big challenge because of different light conditions outdoors, weather condition. Therefore, new illumination invariant techniques must be developed for dog face recognition. After performance evaluation of different face recognition algorithms with different covariates, we hereby conclude that algorithm developers, scientist, different researchers have yet to explore the depths of the process of dog face recognition.

\section{References}

[1] Palika, L. (2004) Purebred Rescue Dog Adaptation: Rewards and Realities. John Wiley \& Sons, Hoboken.

[2] http://www.komonews.com/news/local/New-app-uses-facial-recognition-for-dogs-to-find-lost-pets-225590872.html

[3] Hjalmar, S.K. and Burghardt, T. (2013) Animal Biometrics: Quantifying and Detecting Phenotypic Appearance. 
Trends in Ecology and Evolution, 28, 432-41. http://dx.doi.org/10.1016/j.tree.2013.02.013

[4] Petersen, W.E. (1992) The Identification of the Bovine by Means of Nose-Prints. Journal of Dairy Sciences, 5, 249258. http://dx.doi.org/10.3168/jds.S0022-0302(22)94150-5

[5] Viola, P. and Jones, M. (2004) Robust Real-Time Face Detection. International Journal of Computer Vision, 57, 137-154. http://dx.doi.org/10.1023/B:VISI.0000013087.49260.fb

[6] Gonzales, B.U., Corkery, G., Barry B., Butler, F., McDonnell, K. and Ward, S. (2008) Assessment of Retinal Recognition Technology as a Biometric Method for Sheep Identification. Journal of Computers and Electronics in Agriculture, 60, 156-166. http://dx.doi.org/10.1016/j.compag.2007.07.010

[7] Yamada, A., Kojima, K., Kiyama J., Okamoto, M. and Murata, H. (2011) Directional Edge-Based Dog and Cat Face Detection Method for Digital Camera. Proceedings of International Conference on Consumer Electronics IEEE (ICCE), Las Vegas, 9-12 January 2011, 87-88.

[8] Ketprom, U., et al. (2011) RFID for Cattle Traceability System at Animal Checkpoint. Proceedings of the IEEE Conference on Annual Service Research \&Innovative Institute (SRII), San Jose, March 29 2011-April 2 2011, 517-521.

[9] Parkhi, O.M., Vedaldi, A., Zisserman, A. and Jawahar, C.V. (2012) Cats and Dogs. IEEE Computer Vision and Pattern Recognition, Providence, 16-21 June 2012, 3498-3505.

[10] Chanvichitkul, M., Kumhom, P. and Chamnongthai, K. (2007) Face Recognition Based Dog Breed Classification Using Coarse-to-Fine Concept and PCA. Proceedings of Asia-Pacific Conference on Communications, Bangkok, 18-20 October 2007, 25-29.

[11] Voithi, V.L., et al. (2013) Comparison of Visual and DNA Breed Identification of Dogs and Inter-Observer Reliability. American Journal of Sociological Research, 3, 17-29.

[12] Voith, V.L., et al. (2009) Comparison of Adoption Agency Breed Identification and DNA Breed Identification of Dogs. Journal of Applied Animal Welfare Science, 12, 253-262. http://dx.doi.org/10.1080/10888700902956151

[13] Dogster. http://www.dogster.com/

[14] Flickr. http://www.flicker.com/

[15] Google Images. http://images.google.com

[16] Catster. http://www.catster.com/

[17] Mydogspace. http://www.mydogspace.com/

[18] Turk, M.A. and Pentland, A.P. (1991) Face Recognition Using Eigenfaces. Proceedings of IEEE Computer Vision and Pattern Recognition, Maui, 3-6 June 1991, 586-591.

[19] Zhao, W., Chellappa, R. and Krishnaswamy, A. (1998) Discriminant Analysis of Principal Components for Face Recognition. Proceedings of the International Conference on Face and Gesture Recognition, Nara, 14-16 April 1998, 336-341.

[20] Bartlett, M.S., Movellan Javier, R. and Sejnowski, T.J. (2002) Face Recognition by Independent Component Analysis. IEEE Transactions on Neural Networks, 13, 1450-1464. http://dx.doi.org/10.1109/TNN.2002.804287

[21] Ojala, T., Pietikainen, M. and Maenpaa, T. (2002) Multiresolution Gray-Scale and Rotation Invariant Texture Classification with Local Binary Patterns. IEEE Transactions on Pattern Analysis and Machine Intelligence, 24, 971-987. http://dx.doi.org/10.1109/TPAMI.2002.1017623

[22] Ahonen, T., Hadid, A. and Pietikainen, M. (2006) Face Description with Local Binary Patterns: Application to Face Recognition. IEEE Transactions on Pattern Analysis and Machine Intelligence, 28, 2037-2041. http://dx.doi.org/10.1109/TPAMI.2006.244

[23] Belhumeur, P.N., Hespanha, J.P. and Kriegman, D. (1997) Eigenfaces vs. Fisherfaces: Recognition Using Class Specific Linear Projection. IEEE Transactions on Pattern Analysis and Machine Intelligence, 19, 711-720. http://dx.doi.org/10.1109/34.598228

[24] Zhao, H. and Yuen, P.C. (2008). Incremental Linear Discriminant Analysis for Face Recognition. Systems, Man, and Cybernetics. Part B: IEEE Transactions on Cybernetics, 38, 210-221. http://dx.doi.org/10.1109/TSMCB.2007.908870

[25] Weng, J., Zhang, Y. and Hwang, W.-S. (2003) Candid Covariance-Free Incremental Principal Component Analysis. IEEE Transactions on Pattern Analysis and Machine Intelligence, 25, 1034-1040. http://dx.doi.org/10.1109/TPAMI.2003.1217609

[26] Chang, C.C. and Lin, C.-J. (2001) LIBSVM: A Library for Support Vector Machines. http://www.csie.ntu.edu.tw/ cjlin/libsvm/

[27] Heisele, B., Ho, P. and Poggio, T. (2001) Face Recognition with Support Vector Machines: Global versus Component-Based Approach, Proceedings of 8th IEEE International Conference on Computer Vision (ICCV) Vancouver, 2 , 
688-694.

[28] Guo, G., Li, S.Z. and Chan, K. (2001) Face Recognition by Support Vector Machines. Image and Vision Computing, 19, 631-638. http://dx.doi.org/10.1016/S0262-8856(01)00046-4 